\title{
Polymorphism and superconductivity in the V-Nb-Mo-Al-Ga high-entropy alloys
}

Jifeng $\mathrm{Wu}^{1,2,3}$, Bin $\mathrm{Liu}^{1,2,3}$, Yanwei Cui ${ }^{2,3,4}$, Qinqing $\mathrm{Zhu}^{1,2,3}$, Guorui Xiao ${ }^{2,3,4}$, Hangdong Wang ${ }^{5}$, Siqi $\mathrm{Wu}^{4}$, Guanghan $\mathrm{Cao}^{4}$ and Zhi Ren ${ }^{2,3^{*}}$

\begin{abstract}
High-entropy alloys (HEAs) are the focus of current research for their diverse properties, including superconductivity and structural polymorphism. However, the polymorphic transition has been observed only in nonsuperconducting HEAs mostly under high pressure. Here we report the discovery of the superconductivity and temperature-driven polymorphism in $\left(\mathrm{V}_{0.5} \mathrm{Nb}_{0.5}\right)_{3-x} \mathrm{Mo}_{x} \mathrm{Al}_{0.5} \mathrm{Ga}_{0.5}(0.2 \leq$ $x \leq 1.4)$ HEAs, which are of a single body-centered cubic (bcc) structure for $x=0.2$ and a mixture of the bcc and A15 structures for higher $x$ values. Upon annealing, the bcc structure undergoes a polymorphic transformation to the A15 one and all HEAs exhibit bulk superconductivity. For the sample with $x$ $=0.2$, the bcc polymorph is not superconducting down to $1.8 \mathrm{~K}$, whereas the A15 polymorph has a superconducting transition temperature $T_{\mathrm{c}}$ of $10.2 \mathrm{~K}$ and estimated zero-temperature upper critical field $B_{\mathrm{c} 2}(0)$ of $20.1 \mathrm{~T}$, both of which are the highest among HEA superconductors. With increasing Mo content $x$, both $T_{\mathrm{c}}$ and $B_{\mathrm{c} 2}(0)$ of the A15-type HEAs decrease, yet the large ratio of $B_{\mathrm{c} 2}(0) / T_{c}$ signifies a disorder-induced enhancement of the upper critical field over a wide $x$ range. The decrease in $T_{\mathrm{c}}$ is attributed to the decrease in both the electronic specific-heat coefficient and electron-phonon coupling strength. Furthermore, the valence electron count dependence of $T_{c}$, which is different from both the binary A15 and other structurally different HEA superconductors, suggests that $T_{c}$ may be increased further by reducing the number of valence electrons. Our results not only uncover HEA superconductors of a new structural type, but also provide the first example of polymorphism-dependent superconductivity in HEAs.
\end{abstract}

Keywords: polymorphsim, superconductivity, V-Nb-Mo-Al-Ga high-entropy alloys

\section{INTRODUCTION}

In materials science, polymorphism refers to the property of a solid material to crystallize between at least two distinct structures. For superconductors, the presence of polymorphs is of considerable interest since it offers a unique opportunity to study how the spatial atomic arrangement affects the superconducting properties without changing the chemical composition, which may provide useful clues to the superconducting mechanism [1]. So far, the concurrence of structural polymorphism and superconductivity at ambient pressure has been observed only in ordered materials, such as pure elements [2], binary alloys $[3,4]$, ternary rare/alkali-earth intermetallics $[5,6]$, perovskite oxides [7], doped fullerides [1], transition metal dichalcogenides [8], and organic chargetransfer salts $[9,10]$.

Recently, HEAs have received much attention due to their fascinating mechanical, thermal and physical properties [11-15]. These alloys are single solid-solution phases made up of five or more metal elements, whose concentrations are restricted between $5 \%$ and $35 \%$ atomic percent. Due to the extremely high chemical disorder, HEAs can be viewed as a metallic glass with an ordered lattice, and have been found mostly in high symmetry crystal structures, such as body-centered cubic (bcc), facecentered cubic ( $\mathrm{fcc}$ ), and hexagonal close packing (hcp). Among the large number of HEAs studied, a few members have been reported to show bulk type-II superconductivity [16]. These HEA superconductors are based on $\mathrm{d}$ transition metal elements, and can be categorized into four different structural types: bcc type [17], a-Mn type [18], CsCl type [19], and hcp type [20]. In particular,

\footnotetext{
${ }^{1}$ Department of Physics, Fudan University, Shanghai 200433, China

2 School of Science, Westlake University, Hangzhou 310024, China

${ }^{3}$ Institute of Natural Sciences, Westlake Institute for Advanced Study, Hangzhou 310024, China

${ }^{4}$ Department of Physics, Zhejiang University, Hangzhou 310027, China

${ }^{5}$ Department of Physics, Hangzhou Normal University, Hangzhou 310036, China

*Corresponding author (email: renzhi@westlake.edu.cn)
} 
CsCl-type $(\mathrm{ScZrNb})_{0.65}(\mathrm{RhPd})_{0.35}$ HEA has the highest $T_{\mathrm{c}}$ of $\sim 9.3 \mathrm{~K},[19]$ and the highest $B_{\mathrm{c} 2}(0)$ of $\sim 11.7 \mathrm{~T}$ is achieved in the $(\mathrm{TaNb})_{0.5}(\mathrm{ZrHfTi})_{0.5}$ HEA with a simple bcc structure [21]. Moreover, the bcc-type $(\mathrm{TaNb})_{0.67}$ $(\text { HfZrTi })_{0.33}$ HEA shows robust zero-resistance superconductivity without structural transition at pressures up to $190 \mathrm{GPa}$, demonstrating the potential of HEAs for application under extreme conditions [22]. However, no polymorphs of these HEA superconductors have been reported so far. As a matter of fact, the polymorphic transitions are observed only in nonsuperconducting HEAs and mostly at very high pressure, though the different polymorphs can still exist after the pressure is relieved [23-25].

It is known that the formation energies of the bcc and A15 phases are very similar in some $\mathrm{A}_{3} \mathrm{~B}$-type compounds, where $\mathrm{A}$ is a group VB or VIB transition metal element such as $\mathrm{V}, \mathrm{Nb}$ and $\mathrm{Mo}$, and $\mathrm{B}$ is usually a IIIA main group element such as $\mathrm{Al}$ and $\mathrm{Ga}[3,26,27]$. Consequently, a bcc-to-A 15 polymorphic phase transition can be induced by thermal annealing. It is therefore of interest to study the HEAs based on the V-Nb-Mo-Al-Ga system, which, however, have not been explored to date. In this paper, we present a systematic study on the structural and physical properties of the $\left(\mathrm{V}_{0.5} \mathrm{Nb}_{0.5}\right)_{3-x} \mathrm{Mo}_{x} \mathrm{Al}_{0.5} \mathrm{Ga}_{0.5}$ HEAs for $0.2 \leq x \leq 1.4$. It is shown that, the arc-melted (as-cast) HEA with $x=0.2$ possesses a disordered bcc structure, which transforms to the A15 structure after annealing at $1600^{\circ} \mathrm{C}$. Whereas the bcc polymorph remains normal down to $1.8 \mathrm{~K}$, the $\mathrm{A} 15$ one turns out to be a bulk superconductor with a $T_{\mathrm{c}}$ of $10.2 \mathrm{~K}$ and an orbitally-limited $B_{\mathrm{c} 2}(0)$ of $20.1 \mathrm{~T}$. For $x \geq 0.4$, the as-cast HEAs contain a mixture of bcc and A15 polymorphs, and a similar bcc-to-A15 polymorphic transition is observed upon annealing. With increasing Mo content $x$, both $T_{\mathrm{c}}$ and $B_{\mathrm{c} 2}(0)$ of the A15-type HEAs decrease, while the ratio of $B_{\mathrm{c} 2}(0) / T_{\mathrm{c}}$ for $0.2 \leq x \leq 1.2$ is larger than or comparable to that of $\mathrm{Nb}_{3} \mathrm{Sn}$. The decrease in $T_{\mathrm{c}}$ is concomitant with a decrease in both the density of states at the Fermi level $\left[N\left(E_{\mathrm{F}}\right)\right]$ and electron-phonon coupling strength, as expected in the Bardeen-Cooper-Schreiffer (BCS) theory. By comparing the valence electron account (VEC) dependence of $T_{\mathrm{c}}$ for these A15-type HEAs, the binary A15 and other structurally different HEA superconductors, the immanent mechanism is revealed.

\section{EXPERIMENTAL SECTION}

The V-Nb-Mo-Al-Ga HEAs were prepared by arc melting high-purity V (99.9\%), Nb (99.9\%), Mo (99.9\%), Al (99.9\%) powders and Ga shots (99.9\%) according to the stoichiometric ratio of $\mathrm{V}: \mathrm{Nb}: \mathrm{Mo}: \mathrm{Al}: \mathrm{Ga}=(1.5-x / 2):(1.5$ $-x / 2): x: 0.5: 0.5$ with $x=0.2,0.4,0.6,0.8,1.0,1.2$ and 1.4. In order to minimize the volatilization of $\mathrm{Al}$ and $\mathrm{Ga}$ during the arc melting process, the mixture prereacted at $1000^{\circ} \mathrm{C}$ for one week, and then was melted in an arc furnace under high-purity argon atmosphere. The melts were turned over and remelted several times to ensure homogeneity, followed by rapid cooling on a water-chilled copper plate. For annealing experiments, the as-cast ingots were thoroughly ground and pressed into pellets, which were loaded in alumina crucibles in an argon-filled glove box. The crucibles were then placed in Ta tubes, sealed in evacuated quartz tubes and heated at $1600^{\circ} \mathrm{C}$ under argon atmosphere in a muffle furnace for $12 \mathrm{~h}$. After furnace cooling to $900^{\circ} \mathrm{C}$, the quartz tubes were quenched into the cold water.

The phase purity of as-cast and annealed HEAs was checked by powder X-ray diffraction (XRD) using a Bruker D8 Advance X-ray diffractometer with $\mathrm{Cu} \mathrm{Ka}$ radiation at room temperature. The structural refinements were performed by using the JANA2006 program [28]. The chemical composition was measured with an energy-dispersive X-ray spectrometer (EDX, Model Octane Plus) affiliated to a Zeiss field emission scanning electron microscope. The spectra were collected on different locations of each sample for averaging. The electron diffraction was taken with a JEM-2100F transmission electron microscope operated at an accelerating voltage of $200 \mathrm{kV}$. The electrical resistivity and specific heat were measured by the standard four-probe and the relaxation methods, respectively. The resistivity and specific heat measurements down to $1.8 \mathrm{~K}$ and up to $9 \mathrm{~T}$ were carried out by a Quantum Design PPMS-9 Dynacool. The direct current $(\mathrm{dc})$ magnetization measurements down to $1.8 \mathrm{~K}$ were performed on a commercial SQUID magnetometer (MPMS3).

\section{RESULTS AND DISCUSSION}

The powder XRD patterns at room temperature for the series of as-cast and annealed $\left(\mathrm{V}_{0.5} \mathrm{Nb}_{0.5}\right)_{3-x} \mathrm{Mo}_{x} \mathrm{Al}_{0.5} \mathrm{Ga}_{0.5}$ HEAs are shown in Fig. 1a and b, respectively. In Fig. 1a, for the as-cast HEA with $x=0.2$, only several diffraction peaks appear and can be well indexed to the bcc structure with a lattice constant of $3.163 \AA$. At higher $x$ values, a number of additional peaks arise at $2 \theta$ angles corresponding to the A15-type structure, whose strongest peak overlaps with that of the bcc one. The peak intensity of the A15 structure tends to grow with increasing $x$, yet the coexistence of two phases persists up to $x=1.4$, the highest Mo content investigated. Upon annealing at 


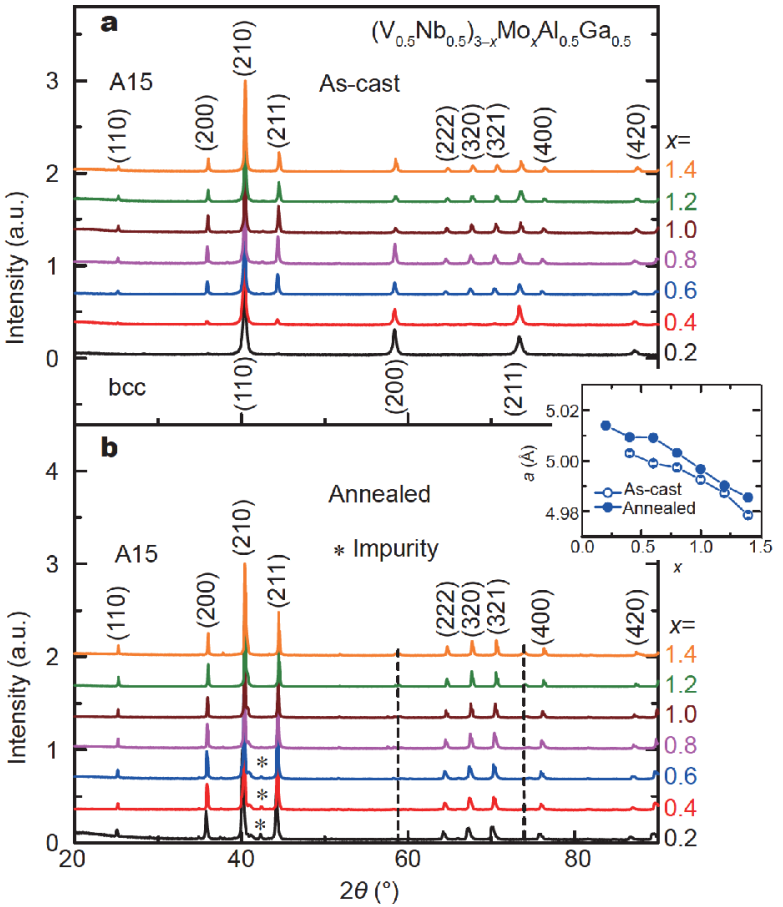

Figure $1(\mathrm{a}, \mathrm{b})$ Powder XRD patterns at room temperature for the ascast and annealed $\left(\mathrm{V}_{0.5} \mathrm{Nb}_{0.5}\right)_{3-x} \mathrm{Mo}_{x} \mathrm{Al}_{0.5} \mathrm{Ga}_{0.5}$ HEAs. The major diffraction peaks for the as-cast HEAs with $x \leq 0.4$ are indexed to the bcc structure. While for $x \geq 0.6$, the major peaks are indexed to the A15 structure. For annealed HEAs, the major peaks are indexed to the A15 structure, and the small impurity peaks for $x \leq 0.6$ are marked by the asterisks. The inset between (a) and (b) shows the lattice parameter of the A15 phase for the as-cast and annealed HEAs plotted as a function of the Mo content $x$.

$1600^{\circ} \mathrm{C}$, the peaks of the bcc structure disappear but those belonging to the A15 structure remain. This reveals that the thermal treatment results in a bcc-to-A15 structural transformation. For $x \leq 0.6$, a small impurity peak is observed at $2 \theta \approx 42^{\circ}$, probably coming from the $\mathrm{Nb}_{2} \mathrm{Al}-$ type sigma phase. By using a least-squares method, the lattice constant of the A15 structure for both the as-cast and annealed HEAs was determined and plotted as a function of the Mo content $x$ in the inset of Fig. 1. One can see that, for $x=0.2$, the $a$-axis is $\sim 5 \AA$, and the corresponding unit-cell volume $\left(\sim 125 \AA^{3}\right)$ is nearly four times that of the bcc polymorph $\left(\sim 31.6 \AA^{3}\right)$. At higher $x$ values, the $a$-axis is $0.1 \%-0.2 \%$ larger in annealed HEAs than in as-cast HEAs. Nevertheless, in both cases, the $a$ axis decreases monotonically with increasing $x$, which means that the unit-cell volume shrinks as more Mo atoms are incorporated. On the other hand, EDX analyses show that the chemical compositions of annealed $\left(\mathrm{V}_{0.5} \mathrm{Nb}_{0.5}\right)_{3-x} \mathrm{Mo}_{x} \mathrm{Al}_{0.5} \mathrm{Ga}_{0.5}$ HEAs are essentially in agreement with the nominal ones, and more importantly, no composition change is observed before and after the annealing process for $x=0.2$ (see Table S1, Supplementary information).

According to the above results, the $\left(\mathrm{V}_{0.5} \mathrm{Nb}_{0.5}\right)_{3-x} \mathrm{Mo}_{x^{-}}$ $\mathrm{Al}_{0.5} \mathrm{Ga}_{0.5}$ HEAs have two polymorphs at ambient pressure: one with the bcc structure and the other with the A15 structure. To gain more insight, we focus on the HEA with $x=0.2$, where the two polymorphs are isolated from each other. Fig. $2 \mathrm{a}$ and $\mathrm{b}$ show the structural refinement profiles for the as-cast and annealed HEAs at this composition based on the $\operatorname{Im} \overline{3} m$ and $P m \overline{3} n$ space groups, respectively, and the refined results are listed in Table 1. In both cases, there is a good agreement between the observed and calculated XRD patterns, as indicated by the low $R_{\mathrm{wp}}$ and $R_{\mathrm{p}}$ values. In the bcc structure, there is only one crystallographic site for the five different atoms, whose occupancies are set by the respective atomic fraction. For the A15 structure, there are two distinct sites $(0.25,0,0.5)$ and $(0,0,0)$, while the atomic occupancy at each site remains unchanged. As a consequence, the onedimensional chains consist of both transition metal and main group element atoms, in contrast to the binary A 15 superconductors [29]. Fig. $2 \mathrm{c}$ and d show the electron diffraction patterns for the bcc and A15 polymorphs, respectively. In the former case, the pattern consists of a series of concentric rings, which match well with the crystal planes of the bcc structure with a small grain size. By contrast, for the A15 one, well defined spots from (100) planes can be observed, which also provides evidence for the growth in grain size during the annealing process. Overall, these results are very similar to those observed in binary $\mathrm{A}_{3} \mathrm{~B}$-type compounds with the bcc and A15 polymorphs [26]. Nevertheless, it should be noted that, for example, annealing $\mathrm{Nb}_{3} \mathrm{Al}$ at $800^{\circ} \mathrm{C}$ for several hours is sufficient to transform the bcc polymorph to the A15 one completely. This heat-treatment temperature is only half of that employed for the present HEAs. Actually, we have also performed annealing experiments at $1000^{\circ} \mathrm{C}$, but found that the transformation is still not complete after more than one week. Hence it appears that, compared with binary compounds, the HEAs indeed have higher thermal stability. Furthermore, since the A15 polymorph can be regarded as a low-temperature phase and remains stable up to $1600^{\circ} \mathrm{C}$, the polymorphic transition from the A15 to bcc structure should occur at a temperature much higher than $1600^{\circ} \mathrm{C}$.

Recently, the effect of $\mathrm{Al}$ alloying on the bcc-type $(\mathrm{TaNb})_{0.67}(\mathrm{ZrHfTi})_{0.33}$ HEA superconductor has been investigated by von Rohr et al. [30]. The results show that the $\left[(\mathrm{TaNb})_{0.67}(\mathrm{ZrHfTi})_{0.33}\right]_{1-x} \mathrm{Al}_{x}$ remains in a bcc struc- 

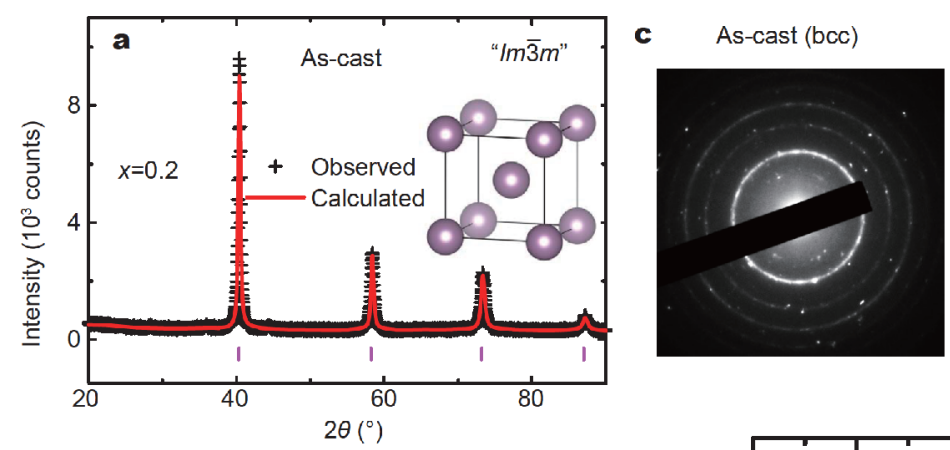

d Annealed (A15)
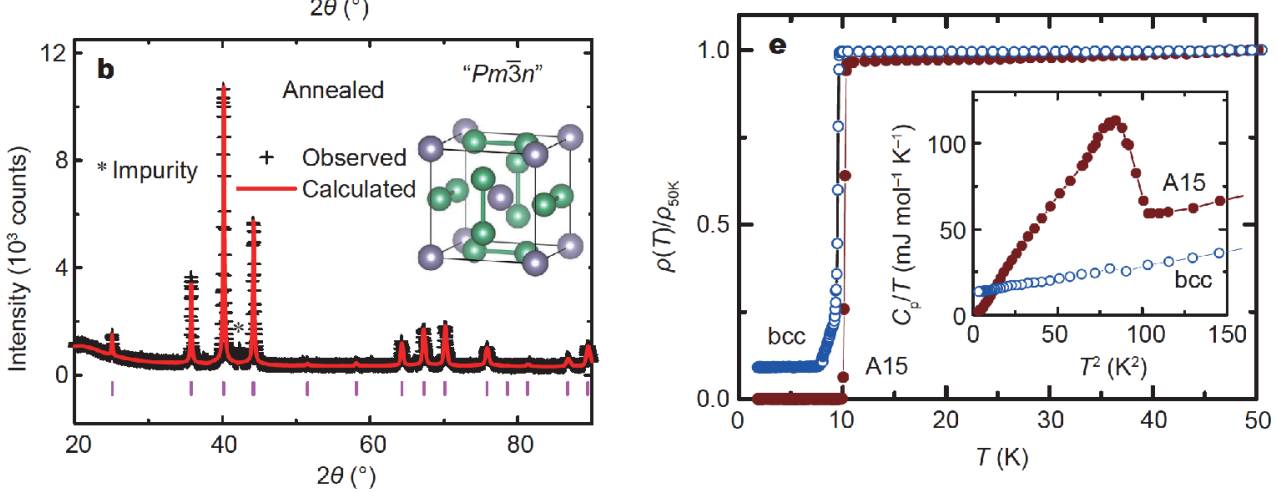

Figure $2(\mathrm{a}, \mathrm{b})$ The structural refinement profiles for the as-cast and annealed $\left(\mathrm{V}_{0.5} \mathrm{Nb}_{0.5}\right)_{3-x} \mathrm{Mo}_{x} \mathrm{Al}_{0.5} \mathrm{Ga}_{0.5} \mathrm{HEAs}$ with $x=0.2$. The small impurity peak in (b) is marked by asterisks. (c, d) Electron diffraction patterns for the bcc and A15 polymorphs, respectively. (e) Temperature dependence of resistivity for the two different polymorphs. The inset shows the corresponding low-temperature specific heat data plotted as $C_{\mathrm{p}} / T$ versus $T^{2}$.

Table 1 Refined crystallographic data of the as-cast and annealed $\left(\mathrm{V}_{0.5} \mathrm{Nb}_{0.5}\right)_{3-x} \mathrm{Mo}_{x} \mathrm{Al}_{0.5} \mathrm{Ga}_{0.5}$ HEAs with $x=0.2$

\begin{tabular}{|c|c|c|c|c|c|c|c|c|}
\hline & \multicolumn{4}{|c|}{ As-cast } & \multicolumn{4}{|c|}{ Annealed } \\
\hline Structural type & \multicolumn{4}{|c|}{ bcc } & \multicolumn{4}{|c|}{ A15 } \\
\hline Space group & \multicolumn{4}{|c|}{$\operatorname{Im} \overline{3} m$} & \multicolumn{4}{|c|}{$P m \overline{3} n$} \\
\hline Lattice parameter & \multicolumn{4}{|c|}{$3.163 \AA$} & \multicolumn{4}{|c|}{$5.014 \AA$} \\
\hline$R_{\mathrm{wp}}$ factor & \multicolumn{4}{|c|}{$6.8 \%$} & \multicolumn{4}{|c|}{$9.3 \%$} \\
\hline$R_{\mathrm{p}}$ factor & \multicolumn{4}{|c|}{$5.3 \%$} & \multicolumn{4}{|c|}{$6.6 \%$} \\
\hline Atoms & $x$ & $y$ & $z$ & Occ. & $x$ & $y$ & $z$ & Occ. \\
\hline $\mathrm{V} 1 / \mathrm{Nb} 1 / \mathrm{Mo} 1 / \mathrm{Al} 1 / \mathrm{Ga} 1$ & 0 & 0 & 0 & $\begin{array}{c}0.35 / 0.35 / 0.05 / 0.125 / \\
0.125\end{array}$ & 0 & 0 & 0 & $\begin{array}{c}0.35 / 0.35 / 0.05 / 0.125 / \\
0.125\end{array}$ \\
\hline $\mathrm{V} 2 / \mathrm{Nb} 2 / \mathrm{Mo} 2 / \mathrm{Al} 2 / \mathrm{Ga} 2$ & & & & & 0.25 & 0 & 0.5 & $\begin{array}{c}0.35 / 0.35 / 0.05 / 0.125 / \\
0.125\end{array}$ \\
\hline
\end{tabular}

ture up to $x=0.3$, but changes to a sigma phase structure with increasing $x$ to 0.4 , suggesting that the structure of this system is instable. Therefore, a polymorphic transition may occur for some $x$ value upon proper thermal treatment. It is worth noting that, even for the Al-free Ta$\mathrm{Nb}-\mathrm{Zr}$-Hf-Ti HEAs, the bcc structure can be unstable against annealing, though no other polymorph has been observed [31]. This suggests that the occurrence of structure polymorphism in HEAs is closely related to their constituent elements.
Fig. 2e shows the temperature dependence of normalized resistivity below $50 \mathrm{~K}$ for the bcc and $\mathrm{A} 15$ polymorphs of the $\left(\mathrm{V}_{0.5} \mathrm{Nb}_{0.5}\right)_{3-x} \mathrm{Mo}_{x} \mathrm{Al}_{0.5} \mathrm{Ga}_{0.5}$ HEA with $x=$ 0.2. A weak temperature dependence is observed in both cases, which is typical for HEAs [17,21]. Cooled below $10.3 \mathrm{~K}$, the resistivity of the A15 polymoprh drops sharply to zero, evidencing a superconducting transition. While a similar resistivity drop is seen at a slightly lower temperature for the bcc polymoprh, no zero resistance is achieved down to $1.8 \mathrm{~K}$. On the other hand, a large spe- 
cific heat $C_{\mathrm{p}}$ jump appears for the A15 polymoprh (see Fig. 2e), confirming the bulk nature of its superconductivity. Nevertheless, there is no anomaly in the $C_{\mathrm{p}}$ data of the bcc counterpart. This suggests that the bulk bcc phase does not superconduct above $1.8 \mathrm{~K}$ and the observed resistive transition originates from a tiny amount of superconducting impurities. In addition, the $C_{\mathrm{p}}$ data indicates that the electronic specific heat coefficient $(\gamma)$ for the bcc polymoprh is much smaller than that of the A15 one, which will be discussed further below. In the next part of the paper, we restrict our attention to the superconducting properties of the A15 polymorph in the annealed $\left(\mathrm{V}_{0.5} \mathrm{Nb}_{0.5}\right)_{3-x} \mathrm{Mo}_{x} \mathrm{Al}_{0.5} \mathrm{Ga}_{0.5}$ HEAs.

Fig. $3 \mathrm{a}-\mathrm{c}$ show the temperature dependence of resisitivity $(\rho)$, magnetic susceptibility $(\chi)$ and $C_{\mathrm{p}}$ of these HEAs below $12 \mathrm{~K}$, respectively. For each $x$ value, the superconducting transition was characterized by a sharp drop in $\rho$, a strong diamagnetic response and a clear $C_{\mathrm{p}}$ jump. Here $T_{c}$, determined as the midpoint of the resistive transition, is found to decrease monotonically from $10.2 \mathrm{~K}$ to $3.2 \mathrm{~K}$ with increasing $x$ to 1.4 . The onset temperature of the diamagnetic signal in the zero-field cooling $\chi_{\mathrm{ZFC}}$ agrees well with the $T_{\mathrm{c}}$ determined from the $\rho$ measurements. Moreover, the $\chi_{\mathrm{ZFC}}$ data at $1.8 \mathrm{~K}$ for all HEAs correspond to a shielding fraction of more than $130 \%$ without correcting the demagnetization factor. This, together with the $C_{\mathrm{p}}$ jump, clearly demonstrates bulk superconductivity in these HEAs. In the normal state, the $C_{\mathrm{p}_{2}}$ data are well fitted by the Debye model $C_{\mathrm{p}} / T$ $=\gamma T+\beta T^{2}$, where $\beta$ is the phononic specific heat coefficient. Once $\beta$ is known, the Debye temperature $\Theta_{\mathrm{D}}$ can be calculated by the formula $\Theta_{\mathrm{D}}=(12 \pi N R / 5 \beta)^{1 / 3}$, where $N$ is the number of atoms per unit cell and $R=$ $8.314 \mathrm{~J} \mathrm{~mol}^{-1} \mathrm{~K}^{-1}$ is the molar gas constant. The analysis results are summarized in Table 2. For $x=0.2, \gamma$ has a value of $30.9 \mathrm{~mJ} \mathrm{~mol}^{-1} \mathrm{~K}^{-2}$ and the normalized specific heat jumps $\Delta \mathrm{C}_{\mathrm{p}} / \gamma T_{\mathrm{c}}$ is estimated to be 2.01 , which is significantly larger than the BCS value of 1.43 [32]. Nevertheless, the jump can be reasonably reproduced by a modified BCS model with $\Delta_{0} / T_{\mathrm{c}}=2.07$, where $\Delta_{0}$ is the fully isotropic gap at $0 \mathrm{~K}$ (see Fig. S1, Supplementary information). This suggests that the superconducting state is still BCS-like [33]. With increasing $x$, the two quantities decrease and reduce to $16.5 \mathrm{~mJ} \mathrm{~mol}^{-1} \mathrm{~K}^{-2}$ and 1.33 at $x=1.4$, respectively. On the other hand, with the knowledge of $T_{\mathrm{c}}$ and $\Theta_{\mathrm{D}}$, the electron-phonon coupling
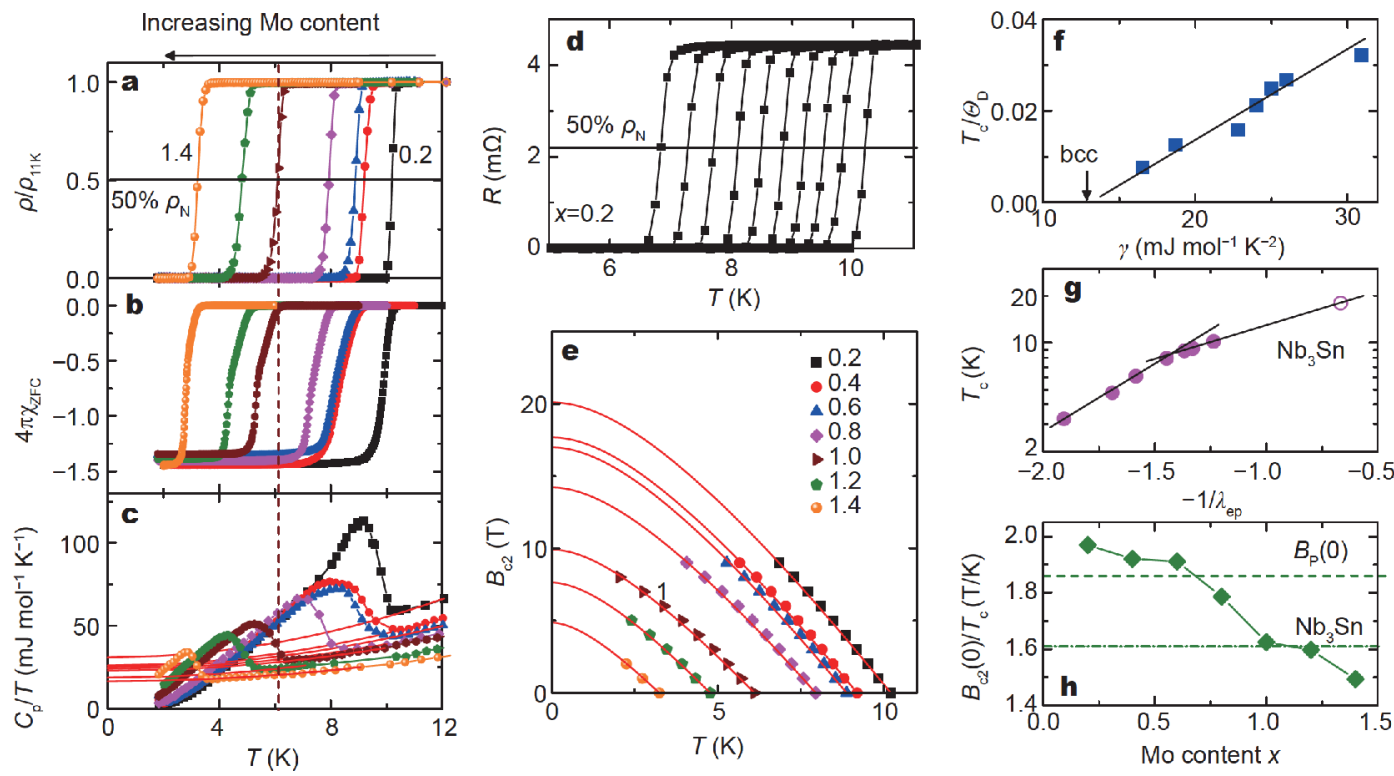

Figure 3 (a-c) Temperature dependence of resistivity, magnetic susceptibility and specific heat below $12 \mathrm{~K}$ for the series of A15-type $\left(\mathrm{V}_{0.5} \mathrm{Nb}_{0.5}\right)_{3-x} \mathrm{Mo}_{x} \mathrm{Al}_{0.5} \mathrm{Ga}_{0.5}$ HEAs. The left-handed arrow indicates the direction of increasing $x$, and the vertical dashed line is a guide to the eyes. In panel (a), the horizontal line denotes the midpoint of the resistive transition. In panel (c), the red solid lines are the fits by the Debye model to the normal-state data. (d) Temperature dependence of resistivity for $x=0.2$ under magnetic fields up to $9 \mathrm{~T}$ in increments of $1 \mathrm{~T}$. The dashed line marks the midpoint of the resistive transition. (e) Temperature dependence of the upper critical fields determined for the series of HEAs. The solid lines are fitted from the WHH model to the data. (f) $T_{\mathrm{c}} / \Theta_{\mathrm{D}}$ plotted as a function of $\gamma$. The solid line is a guide to the eyes, and the arrow marks the $\gamma$ value for the bcc polymorph with $x=0.2$. (g) Logarithm of $T_{\mathrm{c}}$ plotted as a function of $-1 / \lambda_{\mathrm{ep}}$ for the HEAs, together with the data of $\mathrm{Nb}_{3} \mathrm{Sn}$. The solid lines are a guide to the eyes. (h) Mo content $x$ dependence of $B_{\mathrm{c} 2}(0) / T_{\mathrm{c}}$. The dashed and dashed-dotted lines denote the values for the Pauli limiting field $B_{\mathrm{P}}(0) / T_{\mathrm{c}}$ $=1.86 \mathrm{~T} / \mathrm{K}$ and $\mathrm{Nb}_{3} \mathrm{Sn}$, respectively. 
Table 2 Normal-state and superconducting parameters of A15-type $\left(\mathrm{V}_{0.5} \mathrm{Nb}_{0.5}\right)_{3-x} \mathrm{Mo}_{x} \mathrm{Al}_{0.5} \mathrm{Ga}_{0.5} \mathrm{HEAs}$

\begin{tabular}{|c|c|c|c|c|c|c|c|}
\hline & $x=0.2$ & $x=0.4$ & $x=0.6$ & $x=0.8$ & $x=1.0$ & $x=1.2$ & $x=1.4$ \\
\hline$T_{\mathrm{c}}(\mathrm{K})$ & 10.2 & 9.2 & 8.9 & 7.9 & 6.1 & 4.8 & 3.2 \\
\hline$\gamma\left(\mathrm{mJ} \mathrm{mol}{ }^{-1} \mathrm{~K}^{-2}\right)$ & 30.9 & 26.0 & 25.0 & 24.0 & 22.8 & 18.7 & 16.5 \\
\hline$\Delta C_{\mathrm{p}} / \gamma T_{\mathrm{c}}$ & 2.01 & 1.61 & 1.60 & 1.54 & 1.31 & 1.33 & 1.33 \\
\hline$\Theta_{\mathrm{D}}(\mathrm{K})$ & 317 & 342 & 355 & 374 & 383 & 377 & 424 \\
\hline$\lambda_{\mathrm{ep}}$ & 0.81 & 0.75 & 0.73 & 0.69 & 0.63 & 0.59 & 0.52 \\
\hline$B_{\mathrm{c} 2}(0)(\mathrm{T})$ & 20.1 & 17.7 & 17.0 & 14.2 & 9.9 & 7.6 & 4.8 \\
\hline$\xi_{\mathrm{GL}}(\mathrm{nm})$ & 4.0 & 4.3 & 4.4 & 4.8 & 5.8 & 6.6 & 8.3 \\
\hline
\end{tabular}

constant $\lambda_{\text {ep }}$ can be calculated by the inverted McMillan formula [34],

$\lambda_{\text {ep }}=\frac{1.04+\mu^{*} \ln \left(\Theta_{\mathrm{D}} / 1.45 T_{\mathrm{c}}\right)}{\left(1-0.62 \mu^{*}\right) \ln \left(\Theta_{\mathrm{D}} / 1.45 T_{\mathrm{c}}\right)-1.04}$,

where $\mu^{*}$ is the Coulomb repulsion pseudopotential. Assuming that $\mu^{*}=0.13, \lambda_{\text {ep }}$ values are also found to decrease from 0.81 to 0.52 with increasing $x$ from 0.2 to 1.4. Taken together, these results suggest that the annealed $\left(\mathrm{V}_{0.5} \mathrm{Nb}_{0.5}\right)_{3-x} \mathrm{Mo}_{x} \mathrm{Al}_{0.5} \mathrm{Ga}_{0.5}$ HEAs are moderately coupled superconductors for $0.2 \leq x \leq 0.8$ and weakly coupled superconductors for $1 \leq x \leq 1.4$.

To obtain the $B_{\mathrm{c} 2}(0)$ values for these HEAs, temperaturedependent $\rho(T)$ was measured under various magnetic fields up to $9 \mathrm{~T}$, an example of which for $x=0.2$ is shown in Fig. 3d (for other $x$ values see Fig. S2, Supplementary information). With increasing magnetic field, the resistive transition is slightly broadened and shifts toward lower temperatures as expected. The $T_{\mathrm{c}}$ value at different fields is determined based on the same criterion as that under zero field, and the resulting $B_{\mathrm{c} 2}(T)$ phase diagrams are displayed in Fig. 3e. By using the Wathamer-Helfand-Hohenberg $(\mathrm{WHH})$ theory [35], $B_{\mathrm{c} 2}$ can be extrapolated to zero temperature, yielding $B_{\mathrm{c} 2}(0)=20.1,17.7,16.5,14.2,9.9,7.6$ and $4.8 \mathrm{~T}$ for $x=0.2,0.4,0.6,0.8,1.0,1.2$, and 1.4 , respectively. The Ginzburg-Landau (GL) coherence length $\xi_{\mathrm{GL}}$ can be calculated as $\xi_{\mathrm{GL}}=\left(\Phi_{0} / 2 \pi B_{\mathrm{c} 2}(0)\right)^{1 / 2}$, where $\Phi_{0}=$ $2.07 \times 10^{-15} \mathrm{~Wb}$ is the flux quantum. This gives a relatively short $\xi_{\mathrm{GL}}$ varying between 4.0 to $8.3 \mathrm{~nm}$ for these HEAs.

The examination of the correlation between the physical properties for these HEAs reveals the following salient features. First, as shown in Fig. $3 f$, the ratio of $T_{c} /$ $\Theta_{\mathrm{D}}$ increases as the increase of $\gamma$ and hence $N\left(\mathrm{E}_{\mathrm{F}}\right)$, which is consistent with the BCS theory [32]. In this respect, the absence of superconductivity for the bcc polymorph of $x$ $=0.2$ is likely due to the low $N\left(E_{\mathrm{F}}\right)$ since its $\gamma$ value $\left(12.9 \mathrm{~mJ} \mathrm{~mol}^{-1} \mathrm{~K}^{-2}\right.$ ) is only $\sim 40 \%$ of that of the A15 one. Nevertheless, the possibility of a weakening of the electron-phonon coupling strength cannot be excluded. Second, when the logarithm of $T_{c}$ is plotted as a function of the inverted electron-phonon coupling constant $-1 / \lambda_{\text {ep }}$ (see Fig. $3 \mathrm{~g}$ ), two different slopes are discernible, which contrasts with the case of Heusler superconductors [36]. Note that these two slopes correspond well with the weak $(1 \leq x \leq 1.4)$ and moderate $(0.2 \leq x \leq 0.8)$ electronphonon coupling regimes found above, respectively. In addition, the data of the A15 superconductor $\mathrm{Nb}_{3} \mathrm{Sn}$ falls on the extrapolation of the data in the moderate coupling regime. Nevertheless, the slope appears to be steeper for smaller $\lambda_{\text {ep }}$, pointing to a more significant role of electron-phonon coupling on $T_{\mathrm{c}}$ in this regime. Third, as can be seen from Fig. $3 \mathrm{~h}, B_{\mathrm{c} 2}(0) / T_{\mathrm{c}}$ ratio of these HEAs is larger than or comparable to that of $\mathrm{Nb}_{3} \mathrm{Sn}$, despite their much lower $T_{\text {c. }}$ Especially, the $B_{\mathrm{c} 2}(0)$ value for $x \leq 0.6$ slightly exceeds the Pauli paramagnetic limit, $B_{\mathrm{P}}(0)=$ $1.86 T_{\mathrm{c}}$. While this $B_{\mathrm{c} 2}$ behavior has been observed in bcctype $(\mathrm{TaNb})_{0.16}(\mathrm{HfZrTi})_{0.84}$ HEA [21], whose $T_{\mathrm{c}}$ and $B_{\mathrm{c} 2}(0)$ values are only about half of those in the present case. In the dirty limit, we have $B_{\mathrm{c} 2}(0)=-0.693\left(\mathrm{~d} B_{\mathrm{c} 2} / \mathrm{d} T\right)_{T=T_{\mathrm{c}}} T_{\mathrm{c}}$ [35] and $\left(\mathrm{d} B_{\mathrm{c} 2} / \mathrm{d} T\right)_{T=T_{c}} \propto \gamma \rho$ [37], where $\rho_{\mathrm{c}}$ is the normalstate resistivity value just above $T_{c}$. Hence $B_{\mathrm{c} 2}(0) / T_{\mathrm{c}} \propto \gamma \rho_{\mathrm{c}}$. Since the $\gamma$ values of A15-type HEAs are smaller than that of $\mathrm{Nb}_{3} \mathrm{Sn}$ [38], their larger $B_{\mathrm{c} 2}(0) / T_{\mathrm{c}}$ is apparently attributed to an enhancement of $\rho_{\mathrm{c}}$ resulting from the strong disorder, similar to the ball-milled $\mathrm{Nb}_{3} \mathrm{Sn}$ [39]. Also, it is worth noting that the $B_{\mathrm{c} 2}(0) / T_{\mathrm{c}}$ ratio tends to increase with the increase of $T_{c}$, and that a $B_{\mathrm{c} 2}(0)$ value of $\sim 20 \mathrm{~T}$ is attainable for a $T_{\mathrm{c}}$ of $10.2 \mathrm{~K}$. Provided that the $T_{\mathrm{c}}$ can be enhanced to $\sim 15 \mathrm{~K}$, the $B_{\mathrm{c} 2}(0)$ of the A15-type HEAs might be above $30 \mathrm{~T}$, which is higher than that of $\mathrm{Nb}_{3} \mathrm{Sn}$ [40] and may be applied in high field superconducting magnets.

To provide hints for the $T_{\mathrm{c}}$ enhancement, we plot the $T_{\mathrm{c}}$ 


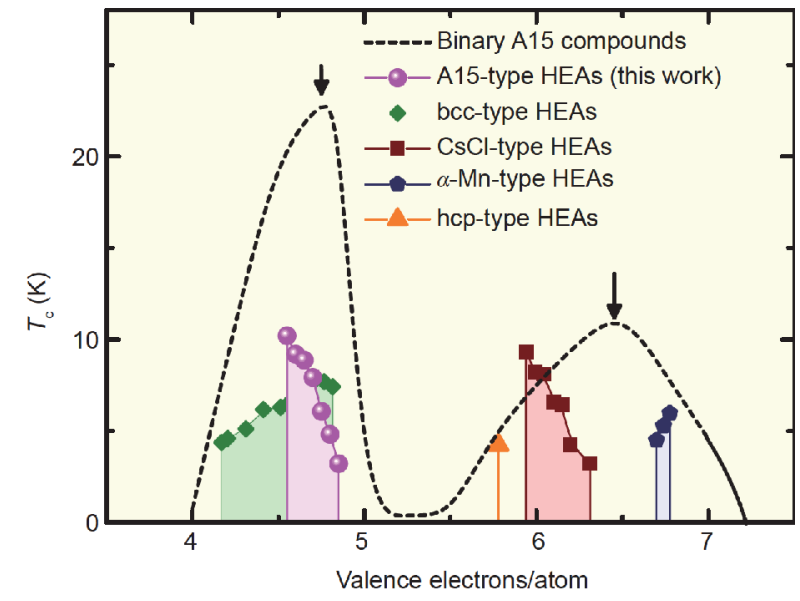

Figure 4 Dependence of $T_{\mathrm{c}}$ on the average number of valence electrons per atom ratio for A15-type $\left(\mathrm{V}_{0.5} \mathrm{Nb}_{0.5}\right)_{3-x} \mathrm{Mo}_{x} \mathrm{Al}_{0.5} \mathrm{Ga}_{0.5}$ HEAs. The data for binary A15 [41] and other structurally different HEA [17-20] superconductors are also included for comparison. The solid lines are a guide to the eyes, and the two $T_{\mathrm{c}}$ maxima for binary A15 compounds are marked by the arrows.

against the average number of valence electrons per atom ratio (e/a) for A15-type $\left(\mathrm{V}_{0.5} \mathrm{Nb}_{0.5}\right)_{3-x} \mathrm{Mo}_{x} \mathrm{Al}_{0.5} \mathrm{Ga}_{0.5}$ HEAs, together with the data of all known binary A15 and other prototype HEA superconductors for comparison, as shown in Fig. $4[16,41]$. As expected by the Matthias rule [42], $T_{\mathrm{c}}$ of the binary A15 superconductors exhibits two maxima around $e / a=4.75$ and 6.5 , respectively. For the A15 HEAs, the e/a values fall between 4.55 and 4.85, suggesting that they still obey the Matthias rule. Nevertheless, their $T_{\mathrm{c}}$ shows a monotonic decrease with increasing e/a. This contrast is not so surprising since the strong disorder inherent to A15 HEAs will smear out $N\left(E_{\mathrm{F}}\right)$ and wipe out its structure that would be in the binary A15 superconductors [43]. Hence the band filling dependence of $T_{c}$ is naturally expected to be different between these two families of materials. In any case, this result suggests that the $T_{\mathrm{c}}$ of A15 HEAs could be further enhanced by reducing $e / a$ to below 4.55. In this regard, replacing Mo with Ta or group IVB elements ( $\mathrm{Ti}, \mathrm{Zr}, \mathrm{Hf}$ ) will be of interest for future studies. On the other hand, it is worth noting that, among all HEA superconductors, only the A15- and bcc-type ones exhibit an overlap of the e/a range, which is consistent with the observation of a polymorphic transition between these two structural types. Nevertheless, the $T_{\mathrm{c}}$ dependence on $e / a$ is quite different for two cases. Hence it appears that, in addition to valence electron count, crystal structure and constituent elements also play a nontrivial role in determining the $T_{\mathrm{c}}$ of HEA superconductors.
Finally, we briefly discuss the implication of our results. First, these results clearly indicate that HEA superconductors are not limited to transition metal elements, which open more possibilities of combinations of elements to compose superconducting HEAs. Second, the A15-type HEAs represent an alternative way of introducing strong disorder to study the effect of atomic ordering on the physical properties of A15 compounds. Previously, such disorder can be introduced by ball milling [44], rapid quenching [45] and high-energy particle radiation [46], all of which have no influence on the chemical composition. In this regard, the chemical complexity in HEAs is expected to provide a fresh insight into the effect of compositional disorder. For example, one question is how this disorder affects normal state resistivity and magnetoresistance, which requires high magnetic fields to suppress superconductivity completely. Third, it is prudent to note that the $\left(\mathrm{V}_{0.5} \mathrm{Nb}_{0.5}\right)_{3-x} \mathrm{Mo}_{x} \mathrm{Al}_{0.5} \mathrm{Ga}_{0.5}$ HEAs exhibit the same polymorphism as the binary combinations of their constituent elements, such as $\mathrm{Nb}_{3} \mathrm{Al}, \mathrm{Mo}_{3} \mathrm{Al}$ and $\mathrm{V}_{3} \mathrm{Ga}$. Further studies are called for to assess the generality of this observation in other polymorphic HEAs.

\section{CONCLUSIONS}

In summary, we have discovered both superconductivity and temperature-driven polymorphism in the $\left(\mathrm{V}_{0.5} \mathrm{Nb}_{0.5}\right)_{3-x} \mathrm{Mo}_{x} \mathrm{Al}_{0.5} \mathrm{Ga}_{0.5}$ HEAs with $x$ in the range of 0.2 to 1.4. The results show that the as-cast HEA has a single bcc structure for $x=0.2$ and a mixture of the bcc and A15 structures for higher $x$ values. Upon annealing, a bcc-to-A 15 polymorphic transition takes place and all HEAs are found to exhibit bulk superconductivity. In particular, for $x=0.2$, though the bcc polymorph is not superconducting down to $1.8 \mathrm{~K}$, the A15 one has a $T_{\mathrm{c}}$ of $10.2 \mathrm{~K}$ and an estimated $B_{\mathrm{c} 2}(0)$ of $20.1 \mathrm{~T}$, both of which are the highest among HEA superconductors. With increasing Mo content $x, T_{\mathrm{c}}$ of these A15-type HEAs decreases monotonically, which is ascribed to the reduction in both electron phonon coupling strength and $N\left(E_{\mathrm{F}}\right)$. Furthermore, the $T_{\mathrm{c}}$ also decreases with increasing e/a from 4.55 to 4.85 . This is different from the binary A15 as well as other structurally different HEA superconductors, and suggests that $T_{\mathrm{c}}$ may be enhanced by reducing the number of valence electrons. On the other hand, the $B_{\mathrm{c} 2}(0) / T_{\mathrm{c}}$ ratio of the A15-type HEAs, which is larger than that of $\mathrm{Nb}_{3} \mathrm{Sn}$ over a broad $x$ range, provides evidence for a disorder-induced enhancement of the upper critical field. Our results not only present a new type of HEA superconductors, but also provide the first example of 
polymorphism-dependent superconductivity in HEAs, which help to understand the interplay between chemical disorder, crystal structure and superconducting properties in these materials.

\section{Received 21 November 2019; accepted 19 December 2019;} published online 10 January 2020

1 Ganin AY, Takabayashi Y, Jeglic $\mathrm{P}$, et al. Polymorphism control of superconductivity and magnetism in $\mathrm{Cs}_{3} \mathrm{C}_{60}$ close to the Mott transition. Nature, 2010, 466: 221-225

2 Petch NJ. a-Tungsten. Nature, 1944, 154: 337

3 Giorgi AL, Matthias BT. Unusual superconducting behavior of the molybdenum-technetium system. Phys Rev B, 1978, 17: 2160-2162

4 Wire MS, Webb GW. Superconductivity and the b.c.c. to A-15 transformation in Nb-Au alloys. J Phys Chem Solids, 1981, 42: 233-238

5 Braun HF, Engel N, Parthé E. Polymorphism and superconductivity of $\mathrm{LaIr}_{2} \mathrm{Si}_{2}$. Phys Rev B, 1983, 28: 1389-1395

6 Xie W, Seibel EM, Cava RJ. The new superconductor $\mathrm{t} P-\mathrm{SrPd}_{2} \mathrm{Bi}_{2}$ : Structural polymorphism and superconductivity in intermetallics. Inorg Chem, 2016, 55: 3203-3205

7 Climent-Pascual E, Ni N, Jia S, et al. Polymorphism in $\mathrm{BaPb}_{1-x} \mathrm{Bi}_{x} \mathrm{O}_{3}$ at the superconducting composition. Phys Rev B, 2011, 83: 174512

8 Luo $\mathrm{H}, \mathrm{Xie} \mathrm{W}$, Tao J, et al. Polytypism, polymorphism, and superconductivity in $\mathrm{TaSe}_{2-x} \mathrm{Te}_{x}$. Proc Natl Acad Sci USA, 2015, 112: E1174-E1180

9 Kikuchi K, Honda Y, Ishikawa Y, et al. Polymorphism and electrical conductivity of the organic superconductor (DMET) ${ }_{2} \mathrm{AuBr}_{2}$. Solid State Commun, 1988, 66: 405-408

10 Rabaça S, Oliveira S, Santos IC, et al. Polymorphism and superconductivity in bilayer molecular metals (CNB-EDT-TTF) $_{4} \mathrm{I}_{3}$. Inorg Chem, 2016, 55: 10343-10350

11 Yeh JW, Chen SK, Lin SJ, et al. Nanostructured high-entropy alloys with multiple principal elements: Novel alloy design concepts and outcomes. Adv Eng Mater, 2004, 6: 299-303

12 Ye YF, Wang Q, Lu J, et al. High-entropy alloy: challenges and prospects. Mater Today, 2016, 19: 349-362

13 Miracle DB, Senkov ON. A critical review of high entropy alloys and related concepts. Acta Mater, 2017, 122: 448-511

14 Zhang W, Liaw PK, Zhang Y. Science and technology in highentropy alloys. Sci China Mater, 2018, 61: 2-22

15 George EP, Raabe D, Ritchie RO. High-entropy alloys. Nat Rev Mater, 2019, 4: 515-534

16 Sun L, Cava RJ. High-entropy alloy superconductors: Status, opportunities, and challenges. Phys Rev Mater, 2019, 3: 090301

17 Koželj P, Vrtnik S, Jelen A, et al. Discovery of a superconducting high-entropy alloy. Phys Rev Lett, 2014, 113: 107001

18 Stolze K, Cevallos FA, Kong T, et al. High-entropy alloy superconductors on an $\alpha-M n$ lattice. J Mater Chem C, 2018, 6: 1044110449

19 Stolze K, Tao J, von Rohr FO, et al. Sc-Zr-Nb-Rh-Pd and Sc-Zr$\mathrm{Nb}$-Ta-Rh-Pd high-entropy alloy superconductors on a CsCl-type lattice. Chem Mater, 2018, 30: 906-914

20 Marik S, Motla K, Varghese M, et al. Superconductivity in a new hexagonal high-entropy alloy. Phys Rev Mater, 2019, 3: 060602

21 von Rohr F, Winiarski MJ, Tao J, et al. Effect of electron count and chemical complexity in the Ta-Nb-Hf-Zr-Ti high-entropy alloy superconductor. Proc Natl Acad Sci USA, 2016, 113: E7144-E7150 22 Guo J, Wang H, von Rohr F, et al. Robust zero resistance in a superconducting high-entropy alloy at pressures up to $190 \mathrm{GPa}$. Proc Natl Acad Sci USA, 2017, 114: 13144-13147

23 Zhang F, Wu Y, Lou H, et al. Polymorphism in a high-entropy alloy. Nat Commun, 2017, 8: 15687

24 Huang EW, Lin CM, Jain J, et al. Irreversible phase transformation in a $\mathrm{CoCrFeMnNi}$ high entropy alloy under hydrostatic compression. Mater Today Commun, 2018, 14: 10-14

25 Wang L, Zhang F, Nie Z, et al. Abundant polymorphic transitions in the $\mathrm{Al}_{0.6} \mathrm{CoCrFeNi}$ high-entropy alloy. Mater Today Phys, 2019, 8: $1-9$

26 Wang R, Dahlgren SD. A microstructural investigation of the bcc to A15 phase transition in sputter-deposited $\mathrm{Nb}_{3} \mathrm{Al}$ superconductors. MTA, 1977, 8: 1763-1768

27 Harada N, Nakano T, Tsuda M, et al. Superconducting properties and rapid heating condition in transformed jelly-roll $\mathrm{Nb} /$ sub 3/Al multifilamentary wires as a function of maximum ohmic-heating temperature. IEEE Trans Appl Supercond, 2001, 11: 3611-3614

28 Petříček V, Dušek M, Palatinus L. Crystallographic computing system JANA2006: General features. Z für KristallographieCrystline Mater, 2014, 229: 345-352

29 Rong-Yao W, Qi-Guang L. Structure of A15 compounds. Phys Stat Sol (A), 1986, 96: 397-406

30 von Rohr FO, Cava RJ. Isoelectronic substitutions and aluminium alloying in the Ta-Nb-Hf-Zr-Ti high-entropy alloy superconductor. Phys Rev Mater, 2018, 2: 034801

31 Vrtnik S, Koželj P, Meden A, et al. Superconductivity in thermally annealed Ta-Nb-Hf-Zr-Ti high-entropy alloys. J Alloys Compd, 2017, 695: 3530-3540

32 Bardeen J, Cooper LN, Schrieffer JR. Theory of superconductivity. Phys Rev, 1957, 108: 1175-1204

33 Johnston DC. Elaboration of the a-model derived from the BCS theory of superconductivity. Supercond Sci Technol, 2013, 26: 115011

34 McMillan WL. Transition temperature of strong-coupled superconductors. Phys Rev, 1968, 167: 331-344

35 Werthamer NR, Helfand E, Hohenberg PC. Temperature and purity dependence of the superconducting critical field, $H_{c 2}$. III. Electron spin and spin-orbit effects. Phys Rev, 1966, 147: 295-302

36 Klimczuk T, Wang CH, Gofryk K, et al. Superconductivity in the Heusler family of intermetallics. Phys Rev B, 2012, 85: 174505

37 Orlando TP, McNiff EJ, Foner S, et al. Critical fields, Pauli paramagnetic limiting, and material parameters of $\mathrm{Nb}_{3} \mathrm{Sn}$ and $\mathrm{V}_{3} \mathrm{Si}$. Phys Rev B, 1979, 19: 4545-4561

38 Jo YJ, Zhou J, Sung ZH, et al. Specific heat of $\mathrm{Nb}_{3} \mathrm{Sn}$ : The case for a single gap. APL Mater, 2014, 2: 106101

39 Cooley LD, Hu YF, Moodenbaugh AR. Enhancement of the upper critical field of $\mathrm{Nb}_{3} \mathrm{Sn}$ utilizing disorder introduced by ball milling the elements. Appl Phys Lett, 2006, 88: 142506

40 Foner S, McNiff Jr. EJ. Upper critical fields of cubic and tetragonal single crystal and polycrystalline $\mathrm{Nb}_{3} \mathrm{Sn}$ in DC fields to 30 Tesla. Solid State Commun, 1981, 39: 959-964

41 Dew-Hughes D. Superconducting A-15 compounds: A review. Cryogenics, 1975, 15: 435-454

42 Matthias BT. Empirical relation between superconductivity and the number of valence electrons per atom. Phys Rev, 1955, 97: 74-76

43 Labbe J. Paramagnetic susceptibility in the $\mathrm{V}_{3} \mathrm{Si}$ type of compounds in the normal state. Phys Rev, 1967, 158: 647-654

44 Di LM, Loeff PI, Bakker H. Atomic disorder in $\mathrm{Nb}_{3}$ Sn during heavy 
mechanical impact. J Less Common Met, 1991, 168: 183-193

Blaugher RD, Hein RE, Cox JE, et al. Atomic ordering and superconductivity in A-15 compounds. J Low Temp Phys, 1969, 1: 539-561

46

Sweedler AR, Cox DE, Moehlecke S. Neutron irradiation of superconducting compounds. J Nucl Mater, 1978, 72: 50-69

Acknowledgements This work was financially supported by the National Key Research \& Development Program of China (2017YFA0303002).

Author contributions $\quad \mathrm{Wu} \mathrm{J}$ conceived the idea, synthesized the samples and did the physical property measurements with the assistance from Liu B, Cui Y, Zhu Q, Xiao G. Wang H helped in the EDX analyses. Wu $S$ and Cao G contributed to the magnetic susceptibility measurements. Ren $\mathrm{Z}$ supervised the project and wrote the paper.

Conflict of interest The authors declare no conflict of interest.

Supplementary information Chemical composition of the HEAs, analysis of the specific heat data for $x=0.2$, and resistivity under various field for $x \geq 0.4$ are available in the online version of the paper.

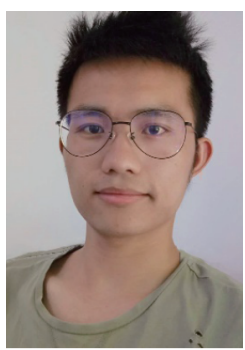

Jifeng $\mathbf{W u}$ is currently a $\mathrm{PhD}$ student in the Department of Physics at Fudan University. He received his bachelor degree from Liaoning Shihua University in 2013, and then received his master degree from Xiamen University in 2016. He worked at GCL Nano for perovskite solar cells, China, from 2016 to 2017. His research interests include the syntheses and characterizations of novel superconductors and supercapacitor materials.

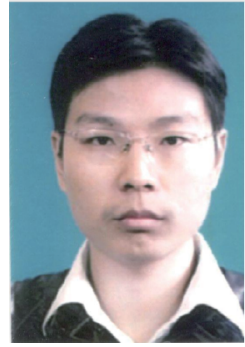

Zhi Ren is currently a research fellow in the School of Science at Westlake University since 2017. He received his bachelor and doctoral degrees from Zhejiang University in 2004 and 2009, respectively. He was a specially appointed researcher in Osaka University from 2009 to 2012 and a postdoctoral assistant in the University of Geneva from 2013 to 2017. His research interests include superconductivity and topological quantum state of materials.

\section{V-Nb-Mo-Al-Ga高熵合金的多晶型性与超导性}

巫继锋 ${ }^{1,2,3}$, 刘斌, 1,3, 崔艳威 ${ }^{2,3,4}$, 祝钦清 ${ }^{1,2,3}$, 肖国锐 ${ }^{2,3,4}$, 王杭栋 ${ }^{5}$, 武思祺 ${ }^{4}$, 曹光旱 ${ }^{4}$, 任之 ${ }^{2,3 *}$

摘要 高熵合金具有结构多晶型性和超导性, 是当前研究的重点. 然而, 多晶型转变仅在非超导的高熵合金中被观察到, 且大多是在 高压条件下. 本文报道了 $\left(\mathrm{V}_{0.5} \mathrm{Nb}_{0.5}\right)_{3-x} \mathrm{Mo}_{x} \mathrm{Al}_{0.5} \mathrm{Ga}_{0.5}(0.2 \leq x \leq 1.4)$ 高熵合金中的超导和温度驱动多晶型性. 实验结果表明当 $x=0.2$ 时 铸态高熵合金具有单一的体心立方 $(\mathrm{bcc})$ 结构, 而当 $x$ 值更高时则为 bcc和A15的混合结构. 经高温退火后, bcc结构向A15结构进行多晶 型转变, 且所有高熵合金均表现出块体超导电性. 对于 $x=0.2$ 的组 分, 其 $\mathrm{bcc}$ 晶型直到 $1.8 \mathrm{~K}$ 仍不具备超导性, 但其 $\mathrm{A} 15$ 晶型却在 $10.2 \mathrm{~K}$ 表现出超导性, 估算零温上临界磁场 $B_{\mathrm{c} 2}(0)$ 为 $20.1 \mathrm{~T}$, 该超导温度 $\left(T_{\mathrm{c}}\right)$ 和磁场强度在已知的高熵合金超导体中均为最高. 随着 $\mathrm{Mo}$ 含 量 $x$ 的增加, $\mathrm{A} 15$ 型高熵合金的 $T_{\mathrm{c}}$ 和 $B_{\mathrm{c} 2}(0)$ 均降低, 但 $B_{\mathrm{c} 2}(0) / T_{\mathrm{c}}$ 比值表 明在宽的 $x$ 范围内存在无序诱导的上临界磁场增加. $T_{\mathrm{c}}$ 的降低归因 于电子比热系数和电声子耦合强度的减小. 此外, 该高熵合金的 $T_{\mathrm{c}}$ 对价电子数依赖关系与二元 A 15 超导体和其他结构高熵合金超导 体均不同, 且表明可以通过降低价电子数目来进一步提高 $T_{\mathrm{c}}$. 本文 不仅揭示了一类新结构类型的高熵合金超导体，而且提供了高熵 合金中依赖于多晶型性超导的首个示例. 